\title{
The differential impact of volatile and intravenous anaesthetics on stress response in the swine
}

\author{
Georgia Kostopanagiotou ${ }^{1}$, Konstantinos Kalimeris ${ }^{1}$, Kalliopi Christodoulaki ${ }^{1}$, \\ Constantinos Nastos ${ }^{1}$, Nikolaos Papoutsidakis ${ }^{1}$, Cleanthi Dima ${ }^{2}$, Charalampos Chrelias ${ }^{3}$, \\ Ageliki Pandazi ${ }^{1}$, Iordanis Mourouzis ${ }^{4}$, Constantinos Pantos ${ }^{4}$
}

12nd Department of Anaesthesiology, ${ }^{2}$ 2nd Department of Clinical Biochemistry, and ${ }^{3} 3 \mathrm{rd}$ Department of Obstetrics and Gynaecology, School of Medicine, University of Athens, Attikon Hospital, ${ }^{4}$ Department of Pharmacology, School of Medicine, University of Athens, Athens, Greece

\begin{abstract}
OBJECTIVE: To compare the effects of various anaesthetics on stress response in the presence and absence of surgical stimulation. DESIGN: Twenty-nine pigs scheduled to undergo surgical central vein catheter placement were randomly allocated to receive only sedation with ketamine-midazolam (group SHAM, $n=5$ ) or general anaesthesia with either propofol $8 \mathrm{mg} / \mathrm{kg} / \mathrm{h}$ (group PROP, $\mathrm{n}=8$ ), sevoflurane 1.0 MAC (group SEVO, $\mathrm{n}=8$ ) or desflurane 1.0 MAC (group DESF, $n=8$ ). Following surgery, anaesthesia was maintained for a total period of 4 hours. Thyroid hormones, noradrenaline, cortisol, corticotrophin (ACTH), $\beta$-endorphin, interleukin- $1 \beta$ and -6 and tumor necrosis factor- $\alpha$ were determined by appropriate methodology after premedication $\left(t_{0}\right)$, after surgical procedure $\left(t_{1}\right)$ and at the end of $4 h\left(t_{2}\right)$. RESULTS: At $t_{1}$ ACTH and $\beta$-endorphin increased in all groups, thyroxin in groups SEVO, DESF and PROP, noradrenaline in group SEVO and cortisol in the SHAM and PROP groups. At $t_{2}$ cortisol and ACTH levels were lower in the PROP compared to SEVO group. Serum cytokines were not significantly altered in either group. CONCLUSIONS: The choice of the general anaesthetic did not affect thyroid hormones levels in either the presence or absence of surgical stimulus. In the presence of surgical stimulation, sevoflurane resulted in higher noradrenaline levels and in higher activity of the ACTH-cortisol axis compared to propofol.
\end{abstract}

Key words: Propofol, Sevoflurane, Stress response, Thyroxin, Cortisol, ACTH endorphins, Anaesthesia

Address for correspondence:

Konstantinos Kalimeris, M.D., 2nd Department of Anaesthesiology, University of Athens School of Medicine, Attikon Hospital, Athens, Greece, 1 Rimini Str., Chaidari, Athens, Greece, Tel: +30-2105826433, Fax: +30-2105326413, Postal Code: 12462, e-mail: k kalimeris@hotmail.com and banesthclin@attikonhospital.gr

Received 19-10-09, Revised 10-12-09, Accepted 20-12-09

\section{INTRODUCTION}

The hormonal and metabolic changes that follow injury or trauma are known as stress response. The hormonal component of surgical stress response principally involves activation of the sympathetic nervous system and alterations in the levels of pi- 
tuitary, thyroid and adrenal hormones. Since stress response during surgical procedures is likely to affect the postoperative clinical outcome, its management may become crucial. ${ }^{1,2}$ Among the factors influencing the intraoperative stress response are the anaesthetic drugs $^{3,4}$ hence, knowledge of their effect on the stress response is very important.

One problem in this regard is that the effects of the anaesthetics per se on the hormonal response are not well known and a clear picture cannot always be obtained. For instance, propofol is known to inhibit sympathetic activation following surgical stimuli, whereas different responses of catecholamines to inhalational agents have been observed. ${ }^{4-6}$ Moreover, the in vitro suppression of adrenal steroidogenesis by propofol has not been confirmed in vivo. ${ }^{7-9}$ Even less clear is the impact of anaesthetic agents on betaendorphin. ${ }^{8-11}$ To further complicate the issue, upon exposure to anaesthetics both increases and decreases of thyroid hormones have been found., ${ }^{3,12-16}$ These diverse results may be due to differences in the study set-up and/or in the study populations, the presence of surgical stress and the varying degrees of surgical stimulation.

The hypothesis behind our study is that general anaesthetics exert a variable effect on the hormonal stress response, depending on whether surgical stimuli are present or not. We therefore aimed to examine whether the type of the anaesthetic used modifies the hormonal responses only during surgical procedure or also during general anaesthesia without surgical manipulations. Ultimately, this could help to clarify which anaesthetic could be chosen in order to obtain a preferred hormonal response in the operating room or in the intensive care unit. In order to highlight possible underlying mechanisms of a diverse effect, we also measured levels of endogenous opioids, interleukins $1 \beta$ and 6 and tumor necrosis factor- $\alpha$ (TNF- $\alpha)$.

\section{EXPERIMENTAL ANIMALS \& METHODOLOGY}

\section{Anaesthesia, Surgery and Monitoring}

The protocol was approved by the Veterinary Directory of the Prefecture of Athens. Care and handling of the animals were in accordance with European guidelines for ethical animal research. Twenty-nine female domestic pigs, weighing 20-24 kg, were used.
After $12 \mathrm{~h}$ deprivation of food and unrestricted access to water, $12 \mathrm{mg}$ midazolam, $50 \mathrm{mg}$ ketamine and $0.5 \mathrm{mg}$ atropine were administered intramuscularly. Intravenous access was established via an auricular vein and general anaesthesia was induced in all animals, except the SHAM animals, with intravenous administration of thiopental sodium $5 \mathrm{mg} / \mathrm{kg}$ and ketamine $2 \mathrm{mg} / \mathrm{kg}$. The trachea was intubated with a cuffed tube of internal diameter $6.0 \mathrm{~mm}$ and mechanical ventilation was started using a volume ventilator (Sulla 808 V, Dräger, Lübeck, Germany) with inspired gases mixture of oxygen and medical air, resulting in inspired concentrations 0.40 for oxygen and 0.60 for nitrogen, tidal volume $=8 \mathrm{ml} / \mathrm{kg}$, and respiratory rate $=10-15$ breaths $/$ min, adjusted to maintain normocapnia. A capnograph (Capnomac Ultima, DatexOhmeda, Helsinki, Finland) for exact measurement of expired concentration of volatile anaesthetics was used. Following iv administration of fentanyl $15 \mu \mathrm{g} / \mathrm{kg}$ and vecuronium bromide $1.5 \mathrm{mg} / \mathrm{kg}$, an $8 \mathrm{~cm}$ incision $2 \mathrm{~cm}$ rightward of the midline, extending from the angle of the mandible to the inner one third of the clavicle, was made. Then, the right external jugular vein was prepared and a central vein catheter $6.5 \mathrm{Fr}$ (Arrow International, Bernville Rd Reading, PA, USA) was inserted, while the right carotid artery was cannulated with a $20 \mathrm{G}$ catheter. The procedure was carried out in all groups; it began immediately after administration of the study anaesthetic and lasted $20 \mathrm{~min}$. A Foley catheter was inserted into the urinary bladder through a small incision of the urinary bladder.

\section{Grouping of animals}

Animals were randomized into four groups by the closed envelope method. Animals in the SHAM group $(n=5)$, after being administered the above premedication, received $1.5 \mathrm{mg} / \mathrm{kg} / \mathrm{h}$ ketamine and $0.25 \mathrm{mg} / \mathrm{kg} / \mathrm{h}$ midazolam by continuous intravenous infusion for four hours in order to achieve sleep and absence of movement together with maintenance of spontaneous breathing without use of a ventilator. During central intravascular catheters and urinary bladder incision for catheter placement, analgesia was achieved via stepwise administration of $0.40 \mathrm{mg}$ fentanyl IV. No other interventions were made in the SHAM animals. Beginning from endotracheal intubation, anaesthesia was maintained for four hours 
in group PROP $(\mathrm{n}=8)$ with continuous intravenous infusion of $8 \mathrm{mg} / \mathrm{kg} / \mathrm{h}$ propofol (Diprivan ${ }^{\circledast}$, AstraZeneca), in the SEVO group $(n=8)$ with 1.0 minimum alveolar concentration (MAC), which is defined as the vapour concentration needed to prevent movement in $50 \%$ of subjects in response to surgical stimulus, this being used as an index of anaesthetic potency to compare anaesthetics of sevoflurane $(2.5 \%$ endexpiratory concentration) and in group $\operatorname{DESF}(\mathrm{n}=8)$ with 1.0 MAC of desflurane (10\% end-expiratory concentration).$^{17}$ All animals except for the SHAM group received vecuronium bromide $0.6 \mathrm{mg} / \mathrm{kg} / \mathrm{h} \mathrm{IV}$ for neuromuscular blockade and additional boluses $10 \mathrm{mg}$ IV were given when spontaneous movements were observed. Analgesia was provided with intravenous infusion of $0.25 \mu \mathrm{g} / \mathrm{kg} / \mathrm{h}$ fentanyl. Dextrose $5 \% 4 \mathrm{ml} / \mathrm{kg} / \mathrm{h}$ and Ringer's lactated $4 \mathrm{ml} / \mathrm{kg} / \mathrm{h}$ were given in order to maintain normoglycemia, normal electrolytic values and central venous pressure between 4 and $7 \mathrm{mmHg}$.

Blood samples were collected in all groups from an auricular vein after premedication $\left(\mathrm{t}_{0}\right)$, immediately after completion of surgical procedure $\left(\mathrm{t}_{1}\right)$ and four hours after beginning of administration of the anaesthetic agent $\left(\mathrm{t}_{2}\right)$. Arterial pressure and heart rate were recorded at the same time-points. Baseline arterial pressure was measured by a sphygmomanometer around the left leg. All animals received euthanasia at the end of the experiment with $3 \mathrm{gr}$ potassium chloride, $100 \mathrm{mg}$ thiopental sodium and $20 \mathrm{mg}$ vecuronium bromide IV.

\section{Hormones \& Cytokines Assays}

Levels of total $\mathrm{T}_{3}$ and $\mathrm{T}_{4}$ in serum were determined by the ACTIVE ${ }^{\circledR}$ Total $\mathrm{T}_{3}$ (DSL-10-3100S) and the ACTIVE $^{\circledR} \mathrm{T}_{4}$ (DSL-10-3200) Enzyme Immunoassay (EIA) kits, respectively, by Diagnostic Systems Laboratories Inc (Webster, Texas, USA). Levels of TSH in serum were determined by the ACTIVE $^{\circledR}$ TSH (DSL-10-5300) Enzyme-Linked Immunosorbent Assay (ELISA) kit by Diagnostic Systems Laboratories Inc. The ELISA scanner for the above measurements was Model A3 by Das (Rome, Italy) and the software used was ACTION 1.8.

Serum noradrenaline was measured using the Noradrenaline EIA kit (BA 10-0200) by Labor Diagnostika Nord GmbH \& Co (Nordhorn, Germany). Serum levels of ACTH and $\beta$-endorphin were determined by the ACTH (EK-001-01) and $\beta$-endorphin (EK022-32) EIA porcine kits by Phoenix Europe GmbH (Karlsruhe, Germany). Levels of IL-1 $\beta$ and IL-6 in serum were determined by the IL-1 $\beta$ (IL01b01) and IL-6 (IL06001) ELISA kits by Orgenium Laboratories (Helsinki, Finland) (typical sensitivity according to manufacturer was $<3 \mathrm{pg} / \mathrm{ml}$ for IL- $1 \beta$ and $<2 \mathrm{pg} / \mathrm{ml}$ for IL-6). Serum TNFa was measured using the TNFa TiterZyme $^{\circledR}$ (900-099) EIA kit by Assay Designs (typical sensitivity was $8.43 \mathrm{pg} / \mathrm{ml}$ ). The microtiter plate reader used for the above assays was Model A3 by Das (Rome, Italy) and the software used was ACTION 1.8. Serum cortisol was measured by the ST AIA-PACK CORT EIA kit by Tosoh Biosciences (Tokyo, Japan), using the IA Tosoh analyzer.

\section{Statistical analysis}

Data are expressed as mean \pm standard deviation. Normal distribution of values was tested with the Kolmogorov-Smirnov test. Mean arterial pressure $(p=0.59)$, heart rate $(p=0.88), P F$ ratio $(p=0.40)$ and arterial carbon dioxide $(\mathrm{p}=0.06)$ values were normally distributed. Regarding hormones, $\mathrm{T}_{3}(\mathrm{p}=0.85), \mathrm{T}_{4}$ $(p=0.74)$, noradrenaline $(p=0.05)$, ACTH $(p=0.11)$ and $\beta$-endorphin $(\mathrm{p}=0.31)$ were normally distributed. Exceptions were TSH $(\mathrm{p}<0.01)$ and cortisol $(\mathrm{p}=0.02)$, which were not normally distributed. Differences within each group for each normally distributed parameter between time-points were analyzed by repeated measures ANOVA and Bonferroni correction was applied for multiple comparisons, except for respiratory parameters which were analyzed by the paired samples $t$-test ( $\alpha$ value corrected according to Bonferroni at .0063). For the non-normally distributed parameters (TSH and cortisol), the differences inside each group for different time-points were tested with Friedman's two-way analysis of variance. Differences between groups for every normally distributed parameter were analyzed by ANCOVA using baseline values as covariate, with the exception of respiratory parameters, which were analyzed with simple ANOVA at both time-points. For the non-normally distributed parameters, the differences between groups were tested with the Kruskal-Wallis test and individual differences were specified with the Mann-Whitney $U$ test. Spearman's coefficient was calculated for the examined correlations. Ob- 
served power of the study was $0.90(\alpha=0.05$, primary outcome $=$ noradrenaline).

\section{RESULTS}

\section{Haemodynamic and respiratory measurements}

Mean arterial pressure and heart rate were constant in all groups, without significant differences among anaesthesia groups. The ratio of arterial partial pressure of oxygen to the inspired fraction of oxygen $(0.40$ in mechanically ventilated and 0.21 in spontaneously breathing animals) also did not differ within or between different anaesthetics groups. Arterial partial pressure of $\mathrm{CO}_{2}$ did not differ among groups; however, mild, but non-significant, hypercapnia was observed at $t_{2}$ in the SHAM animals (Table 1 ).

\section{Thyroid hormones}

Levels of $\mathrm{T}_{3}, \mathrm{~T}_{4}$ or TSH did not differ between groups at any time-point. The levels of $\mathrm{T}_{3}$ decreased significantly only from $t_{1}$ to $t_{2}$ in the propofol group. Levels of $\mathrm{T}_{4}$ increased at $\mathrm{t}_{1}$ in the SEVO, DESF and PROP groups and returned to baseline levels at the end of anaesthesia. $\mathrm{T}_{4}$ levels did not change in the SHAM group. TSH levels as well as the $T_{3} / T_{4}$ ratio did not change with any anaesthetic used (Table 2, Figure 1). Furthermore, none of the thyroid hormones correlated to ACTH, cortisol, noradrenaline or betaendorphin levels.
Nor-adrenaline, cortisol, $A C T H, \beta$-endorphin (Table 3, Figure 1)

The levels of serum noradrenaline increased at $\mathrm{t}_{1}$ only in group SEVO and were significantly higher compared to group PROP. Noradrenaline levels did not significantly change in the other groups.

Cortisol levels increased at $t_{1}$ in the SHAM and PROP groups and returned to baseline values at $t_{2}$. Cortisol concentrations at $t_{2}$ in group PROP did not differ from baseline levels and were significantly lower compared to group SEVO.

ACTH levels at $t_{1}$ were higher from baseline values in all groups. However, ACTH levels remained elevated at $\mathrm{t}_{2}$ only in group SEVO, being significantly higher compared to group PROP.

Beta-endorphin levels increased significantly in all groups at both $t_{1}$ and $t_{2}$ and were positively correlated with ACTH levels ( $r=+0.563$, $\mathrm{p}<0.001)$, with no difference between groups.

\section{Cytokines}

No significant alterations in the serum IL-1 $\beta$, IL-6 and TNF- $\alpha$ were detected in our animals throughout the study period.

\section{DISCUSSION}

Our results indicate that thyroid hormones levels

Table 1. Hemodynamic and respiratory parameters at the various time-points of the study

\begin{tabular}{lccccc}
\hline & Time-point & SHAM & SEVO & DESF & PROP \\
\hline MAP $(\mathbf{k P a})$ & $\mathrm{t}_{0}$ & $13.7 \pm 1.5$ & $12.9 \pm 1.7$ & $12 \pm 2.8$ & $14.8 \pm 3.5$ \\
& $\mathrm{t}_{1}$ & $14.3 \pm 2.1$ & $12 \pm 1.7$ & $11.6 \pm 2.5$ & $14.4 \pm 4.3$ \\
& $\mathrm{t}_{2}$ & $13.2 \pm 1.6$ & $11.7 \pm 1.3$ & $11.9 \pm 2.1$ & $13.3 \pm 3.5$ \\
$\mathbf{H R}(\mathbf{b p m})$ & $\mathrm{t}_{0}$ & $104 \pm 12$ & $99 \pm 16$ & $104 \pm 18$ & $106 \pm 18$ \\
& $\mathrm{t}_{1}$ & $112 \pm 17$ & $91 \pm 15$ & $110 \pm 23$ & $100 \pm 17$ \\
& $\mathrm{t}_{2}$ & $108 \pm 30$ & $103 \pm 11$ & $116 \pm 19$ & $90 \pm 17$ \\
$\mathbf{P} / \mathbf{F}$ ratio $(\mathbf{k P a})$ & $\mathrm{t}_{1}$ & $56 \pm 6$ & $55 \pm 8$ & $50 \pm 11$ & $50 \pm 7$ \\
& $\mathrm{t}_{2}$ & $56 \pm 13$ & $50 \pm 5$ & $48 \pm 12$ & $51 \pm 21$ \\
PaCO $_{2}(\mathbf{k P a})$ & $\mathrm{t}_{1}$ & $5.7 \pm 0.4$ & $4.6 \pm 0.5$ & $5.1 \pm 0.4$ & $4.6 \pm 0.8$ \\
& $\mathrm{t}_{2}$ & $6.1 \pm 0.4$ & $5.3 \pm 0.8$ & $5.3 \pm 0.5$ & $5.5 \pm 0.7$ \\
\hline
\end{tabular}

$\mathrm{HR}$, heart rate; MAP, mean arterial pressure; $\mathrm{PaCO}_{2}$, arterial partial pressure of carbon dioxide; $\mathrm{P} / \mathrm{F}$ ratio, ratio of arterial partial pressure of oxygen to inspired fraction of oxygen; $\mathrm{t}_{0}$, immediately after premedication; $\mathrm{t}_{1}, 20 \mathrm{~min}$ after beginning of administration of the anaesthetic agent; $t_{2}, 4$ hours after beginning of administration of the anaesthetic agent.

Values represent mean \pm SD. SEVO: sevoflurane; DESF: desflurane; PROP: propofol. 
Table 2. Thyroid hormones at the various time-points of the study

\begin{tabular}{|c|c|c|c|c|c|}
\hline & Time-point & SHAM & SEVO & DESF & PROP \\
\hline \multirow[t]{3}{*}{$\mathrm{T}_{3}(\mathrm{ng} / \mathrm{dl})$} & $\mathrm{t}_{0}$ & $86.33 \pm 5.70$ & $85.28 \pm 12.55$ & $70.20 \pm 18.02$ & $72.35 \pm 13.47$ \\
\hline & $\mathrm{t}_{1}$ & $86.61 \pm 5.17$ & $88.11 \pm 17.28$ & $74.63 \pm 24.75$ & $78.04 \pm 17.57$ \\
\hline & $\mathrm{t}_{2}$ & $79.55 \pm 17.97$ & $80.48 \pm 9.63$ & $60.75 \pm 11.02$ & $62.56 \pm 16.73^{\dagger(.014)}$ \\
\hline \multirow[t]{3}{*}{$\mathrm{T}_{4}(\mu \mathrm{g} / \mathrm{dl})$} & $\mathrm{t}_{0}$ & $5.53 \pm 2.51$ & $6.32 \pm 1.32$ & $5.83 \pm 1.93$ & $5.78 \pm 1.51$ \\
\hline & $\mathrm{t}_{1}$ & $6.49 \pm 2.88$ & $7.34 \pm 1.40^{* *}(.001)$ & $6.69 \pm 1.88^{*(.023)}$ & $6.59 \pm 1.74^{* *}(.002)$ \\
\hline & $\mathrm{t}_{2}$ & $6.84 \pm 3.01$ & $5.86 \pm 1.21^{\dagger(.025)}$ & $5.76 \pm 2.08^{\dagger(.032)}$ & $5.61 \pm 1.93$ \\
\hline \multirow[t]{3}{*}{ TSH (mU/L) } & $\mathrm{t}_{0}$ & $0.34 \pm 0.12$ & $0.55 \pm 0.45$ & $0.29 \pm 0.11$ & $1.23 \pm 2.34$ \\
\hline & $\mathrm{t}_{1}$ & $0.33 \pm 0.12$ & $0.49 \pm 0.43$ & $1.50 \pm 2.72$ & $1.25 \pm 2.39$ \\
\hline & $\mathrm{t}_{2}$ & $0.29 \pm 0.12$ & $0.57 \pm 0.45$ & $0.29 \pm 0.12$ & $1.22 \pm 2.29$ \\
\hline \multirow{3}{*}{$\begin{array}{l}T_{3} / T_{4} \text { ratio } \\
\text { (values multiplied by } 1000 \text { ) }\end{array}$} & $\mathrm{t}_{0}$ & $18.67 \pm 10.31$ & $14.25 \pm 5.08$ & $12.37 \pm 2.81$ & $13.05 \pm 3.29$ \\
\hline & $\mathrm{t}_{1}$ & $15.73 \pm 8.26$ & $12.75 \pm 5.41$ & $10.53 \pm 2.18$ & $12.37 \pm 3.37$ \\
\hline & $\mathrm{t}_{2}$ & $14.89 \pm 2.87$ & $14.40 \pm 4.43$ & $10.31 \pm 4.88$ & $12.65 \pm 6.16$ \\
\hline
\end{tabular}

$\mathrm{T}_{3}$, triiodothyronine; $\mathrm{T}_{4}$, thyroxin; $\mathrm{TSH}$, thyroid-stimulating hormone; ${ }^{*}, \mathrm{p}<0.05$ compared to time-point 0 within the group; ${ }^{* *}, \mathrm{p}<0.01$ compared to time-point $\mathrm{t}_{0}$ within the group; $\dagger, \mathrm{p}<0.05$ compared to time-point $\mathrm{t}_{1}$ within the group; $\mathrm{t}_{0}$, immediately after premedication; $\mathrm{t}_{1}, 20$ min after the administration of the anaesthetic agent and at the end of surgery; $\mathrm{t}_{2}, 4$ hours after starting the anaesthetic agent. Values represent mean \pm SD. Values inside parentheses represent exact p values. DESF: desflurane; PROP: propofol; SEVO: sevoflurane. (To convert to SI units mulptiply by 12,87 for $\mathrm{T}_{4}$ values and by 0.015 for $\mathrm{T}_{3}$ values).

Table 3. Serum levels of the stress hormones at the various time-points of the study

\begin{tabular}{|c|c|c|c|c|c|}
\hline & Time-point & SHAM & SEVO & DESF & PROP \\
\hline \multirow[t]{3}{*}{$\mathrm{NA}(\mathrm{ng} / \mathrm{ml})$} & $\mathrm{t}_{0}$ & $1.02 \pm 0.42$ & $1.05 \pm 0.40$ & $0.90 \pm 0.42$ & $0.92 \pm 0.36$ \\
\hline & $\mathrm{t}_{1}$ & $1.28 \pm 0.45$ & $1.95 \pm 0.77^{(*, .019)(\dagger, .002)}$ & $1.25 \pm 0.57$ & $0.86 \pm 0.22$ \\
\hline & $\mathrm{t}_{2}$ & $1.59 \pm 0.90$ & $1.30 \pm 1.22$ & $1.12 \pm 0.76$ & $1.17 \pm 0.74$ \\
\hline \multirow[t]{3}{*}{ Cortisol $(\mu \mathrm{g} / \mathrm{dl})$} & $\mathrm{t}_{0}$ & $3.32 \pm 0.52$ & $2.69 \pm 0.96$ & $3.03 \pm 1.08$ & $2.46 \pm 1.11$ \\
\hline & $\mathrm{t}_{1}$ & $6.45 \pm 0.93$ & $4.16 \pm 2.28$ & $4.70 \pm 2.77$ & $5.68 \pm 3.18^{(\#, .002)}$ \\
\hline & $\mathrm{t}_{2}$ & $2.82 \pm 1.27$ & $8.12 \pm 5.19^{(\#, .039)(\dagger, .006)}$ & $6.39 \pm 2.97^{(\dagger, .019)}$ & $2.01 \pm 2.30$ \\
\hline \multirow[t]{3}{*}{ ACTH (ng/ml) } & $\mathrm{t}_{0}$ & $5.91 \pm 1.08$ & $5.91 \pm 1.98$ & $7.10 \pm 2.86$ & $4.79 \pm 1.94$ \\
\hline & $\mathrm{t}_{1}$ & $13.32 \pm 1.85^{* *}(.003)$ & $13.58 \pm 7.08^{*}(.018)$ & $11.94 \pm 2.45^{*}(.035)$ & $9.18 \pm 3.31^{* *}(.001)$ \\
\hline & $\mathrm{t}_{2}$ & $11.37 \pm 3.45$ & $14.76 \pm 8.16^{(*, .047)(\dagger \dagger, .045)}$ & $9.34 \pm 2.31$ & $7.48 \pm 3.81$ \\
\hline \multirow[t]{3}{*}{$\beta$-END (ng/ml) } & $\mathrm{t}_{0}$ & $0.367 \pm 0.133$ & $0.295 \pm 0.197$ & $0.309 \pm 0.117$ & $0.360 \pm 0.137$ \\
\hline & $\mathrm{t}_{1}$ & $0.804 \pm 0.288^{*}(.023)$ & $0.899 \pm 0.494^{* *}(.006)$ & $1.019 \pm 0.514 *(.010)$ & $0.880 \pm 0.445^{* *}(.008)$ \\
\hline & $\mathrm{t}_{2}$ & $0.886 \pm 0.354^{*(.020)}$ & $0.852 \pm 0.289^{* *}(.002)$ & $0.858 \pm 0.330^{* *}(.006)$ & $0.615 \pm 0.145^{*}(.012)$ \\
\hline
\end{tabular}

ACTH, adrenocorticotropic hormone; $\beta$-END, $\beta$-endorphin; NA, noradrenaline; $(\dagger), p<0.01$ of groups SEVO or DESF compared to group PROP; $(\dagger), \mathrm{p}<0.05$ of group SEVO compared to group PROP; \#, p<0.05 within group; *, p <0.05 compared to timepoint 0 within the group; **, $\mathrm{p}<0.01$ compared to time-point $\mathrm{t}_{0}$ within the group; $\mathrm{t}_{0}$, immediately after premedication; $\mathrm{t}_{1}, 20$ min after starting the anaesthetic agent and at end of surgery; $\mathrm{t}_{2}, 4$ hours after starting the anaesthetic agent. Exact $p$ values for statistically significant differences are shown inside parentheses. Values are presented as mean \pm SD. SEVO: sevoflurane; DESF: desflurane; PROP: propofol. (To convert to SI units mupltiply by 27.59 for cortisol values and by 0.22 for ACTH values).

are not related to the type of anaesthetic in both the presence and absence of surgical stimulation. Sevoflurane caused a greater intraoperative increase in noradrenaline compared to propofol, with no dif- ferences compared to desflurane. Interestingly, the different response of noradrenaline to sevoflurane vanished in the absence of surgical stimulus. In contrast, no difference was observed in the intraoperative 

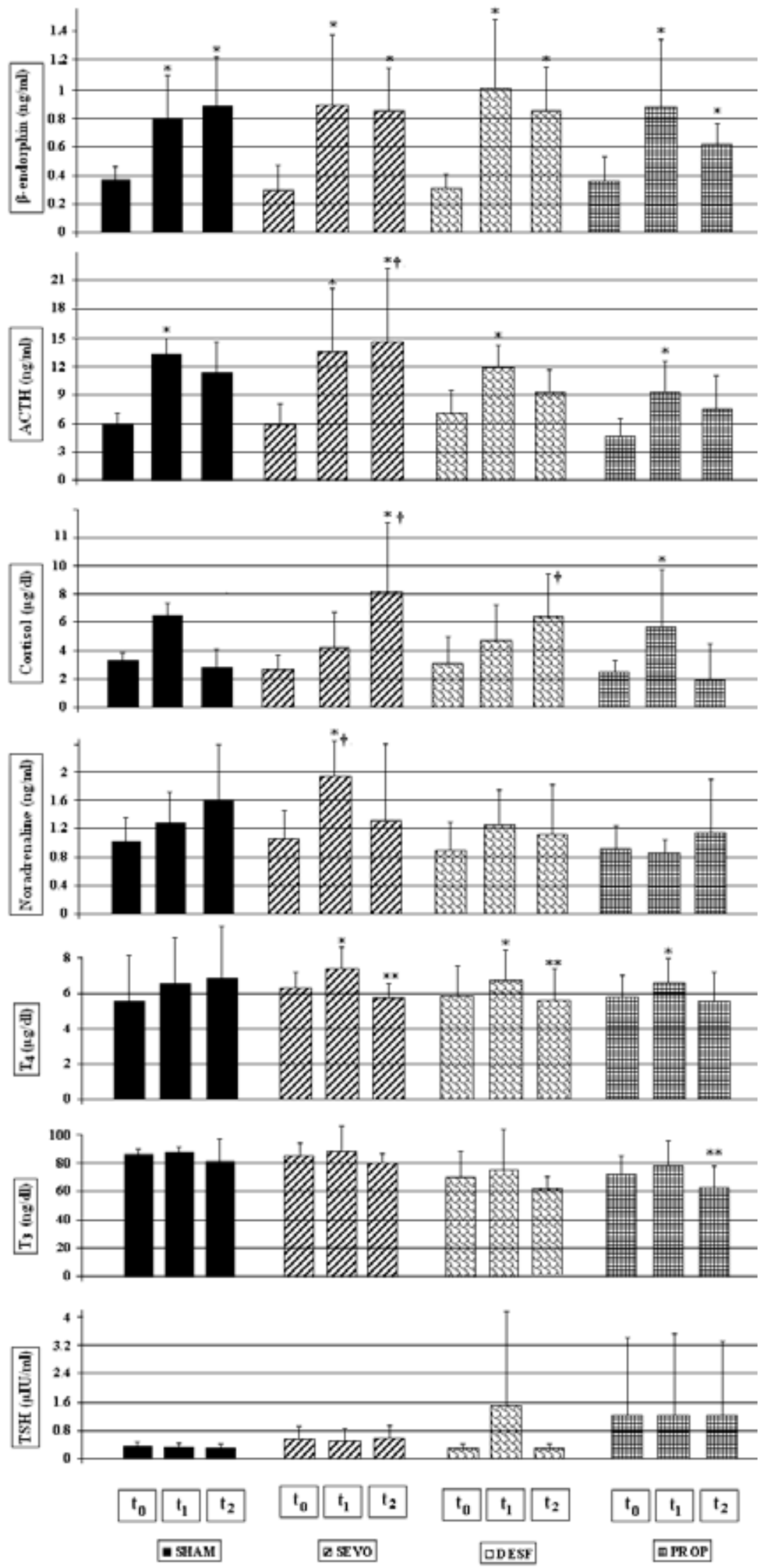

Figure 1. Serum levels of $\beta$-endorphin, adrenocorticotropic hormone (ACTH), noradrenaline, cortisol, thyroid stimulating hormone (TSH), $\mathrm{T}_{3}$ and $\mathrm{T}_{4}$ in the four groups. SHAM, sham group; SEVO, sevoflurane group; DESF, desflurane group; PROP, propofol group, immediately after premedication $\left(\mathrm{t}_{0}\right), 20$ min after starting the anaesthetic agent, and at end of surgery $\left(\mathrm{t}_{1}\right)$, and four hours after starting the anaesthetic agent $\left(\mathrm{t}_{2}\right) .{ }^{*}, \mathrm{p}<0.05$ compared to $\mathrm{t}_{0}$ within the same group; ${ }^{* *}, p<0.05$ compared to $t_{1}$ within the same group; $\dagger, \mathrm{p}<0.05$ of groups SEVO or DESF, as indicated, compared to group PROP for the same time-point. (To convert to SI units mulptiply by 12,87 for $\mathrm{T}_{4}$ values and by 0.015 for $T_{3}$ values). 
cortisol and ACTH responses between anaesthetics. Nevertheless, significantly higher values of ACTH and cortisol were seen in the SEVO group compared to the PROP group at 240 min of general anaesthesia. The Beta-endorphin increase was sustained in all groups and was not differentially affected by the various anaesthetics.

The increase of thyroxin following surgical stimulus was transient and further exposure to general anaesthesia without surgical stimulation restored thyroxin to baseline levels. Some reports refer to either halothane or enflurane as anaesthetic agents that increase $T_{4}$ levels intraoperatively. ${ }^{3,13,14}$ The mechanism involved is not apparent. In our study, $\mathrm{T}_{4}$ levels in the SHAM group did not significantly increase after the surgical stimulus. Since endotracheal intubation is a well known potent stimulus, endotracheal intubation may have played an important role in the transient $T_{4}$ increase. ${ }^{18}$ The restoration of $\mathrm{T}_{4}$ to baseline values after $4 \mathrm{~h}$ of general anaesthesia possibly excludes a clinically significant role of mechanical ventilation and general anaesthetics in thyroid hormones changes.

TSH does not seem to have driven the $\mathrm{T}_{4}$ changes since its levels remained constant. The insignificant alterations of cytokines (interleukins $1 \beta$ and 6 and TNF- $\alpha$ ) probably excludes a systemic effect of the transient thyroid hormones changes, as has been suggested. ${ }^{12}$ We did not find any correlation of the thyroid hormones with noradrenaline, cortisol or ACTH, in accordance with previous studies ${ }^{3}$. A possible role of beta-endorphin in regulating thyroid status at the hypothalamic level has also been suggested, though recent research has questioned such a role..$^{19,20}$ Serum beta-endorphin did not correlate with thyroid hormones in our study, remaining both at $t_{1}$ and $t_{2}$ timepoints higher than baseline values, even when $\mathrm{T}_{4}$ levels had returned to pre-anaesthesia levels. Gerard et al. have also reported no effect of different anaesthetics on beta-endorphin concentrations. ${ }^{21}$ The peripheral conversion of $T_{4}$ to $T_{3}$ was not affected, since the $T_{3} / T_{4}$ ratio did not change during anaesthesia in all groups. These results suggest that the rise of $T_{4}$ in response to endotracheal intubation and surgery may not be due to hypothalamic or pituitary but rather to peripheral mechanisms, which, however, were not significantly modified by the anaesthetics used. Such mechanisms could be inter-compartmental shift or binding proteins alterations, as has been suggested. ${ }^{3}$

Noradrenaline was released in response to the surgical stimulus only in group SEVO, whereas propofol and desflurane did not show such an effect. In agreement with our results, propofol has been shown to inhibit the sympathoadrenal system responses compared both to inhalational and intravenous anaesthetics. ${ }^{4,5,18}$ The attenuation of sympathetic reaction to stimuli by propofol, in conjunction with the lack of effect on basal catecholamine levels, could be of specific benefit not only to surgical patients in whom sympathoadrenal activation would be detrimental but also to patients requiring intensive care and/or sedation postoperatively. Inversely, inhalational anaesthetics could benefit patients who compensate for cardiovascular instability with enhanced sympathetic activity.

Cortisol and ACTH levels differed between anaesthesia groups only when the surgical stimulus was removed. Although after a period without surgical stimuli cortisol concentrations in group PROP were lower compared to group SEVO, they did not differ from baseline values. Reduction in cortisol levels has been documented both in anaesthetized and intensive care patients receiving propofol and in vitro findings suggest a direct inhibitory action of propofol on adrenal steroidogenesis. ${ }^{7,22,23}$ However, more recent studies argue that propofol inhibits cortisol release to stimulatory insults. ${ }^{8,9,24}$ In agreement with the latter studies, cortisol values in the propofol group increased at the end of surgery and returned to baseline values at $4 \mathrm{hrs}$, being at that time lower than in the SEVO and DESF groups. This finding shows that after an initial stimulus, propofol restores cortisol and ACTH levels, at least more rapidly than sevoflurane. Nonetheless, it is possible that these changes depend on both duration of exposure and total dosing of the anaesthetics. This issue could be further addressed by appropriate dose-response experiments.

The changes in noradrenaline and cortisol concentrations were accompanied by increases in $\mathrm{ACTH}$ and beta-endorphin levels, which ran in parallel, showing a significant correlation to each other. This could be attributed to a stimulation of pro-opiomelanocortin, which gives rise to both hormones. However, the secretion of the derivative peptides of pro-opiomelanocortin 
is differentially regulated. ${ }^{25}$ In our study there is the possibility that ketamine used for premedication induced the sustained increase of beta-endorphin, since it has been demonstrated that ketamine directly stimulates its secretion. ${ }^{26}$ After the initial increase in all groups, ACTH levels remained higher in group SEVO compared to group PROP, as did cortisol. Thus, higher cortisol levels in group SEVO could have resulted from a greater activation of pituitary secretion of ACTH in group SEVO compared to group PROP, as has been suggested. ${ }^{27,28}$ Consistent with the observations of Van Hemelrijck et al. and Miyawaki et al., propofol did not suppress either ACTH or cortisol secretion. ${ }^{8,29}$ The initial increase in cortisol seems to have resulted in a proper feedback mechanism in group PROP, reducing ACTH levels at $t_{2}$. In contrast, the sustained increase of ACTH in group SEVO could have resulted from impaired feedback mechanism or from a constant stimulus exerted by SEVO to the pituitary. Further studies are needed to clarify these effects.

\section{Limitations of the study}

A common problem in anaesthesia research is the creation of a true control group, since surgery or mechanical ventilation cannot be achieved without anaesthesia. With this limitation, we chose the use of sedatives without mechanical ventilation in sham animals in order to form an anaesthesia group with lighter depth of anaesthesia. Sevoflurane and desflurane were given in equipotent doses; this may not be applicable to propofol, though haemodynamics do not differ between the three groups. Desflurane has sympathomimetic effects, propofol causes bradycardia and hypotension, while sevoflurane in clinical doses is characterized by cardiovascular stability. The variable haemodynamic properties of the three anaesthetics themselves do not allow a) the conclusion that the doses were equipotent or b) the characterization of their doses as equipotent, when haemodynamics during anaesthesia do not differ. Therefore, comparison of the results must be applied to the propofol group with caution. Another limitation is that premedication with midazolam/ketamine and induction of anaesthesia with thiopental/ketamine could have affected the stress response to surgery. Although the drugs and doses used were identical among the various groups, careful interpretations should be made when comparing studies using different anaesthetic techniques and drugs. Finally, we chose the procedure to be cervical vessels preparation so that the sham animals would have a surgical stimulus, as did the other groups, which could be tolerated by sedated animals. Besides, central lines were deemed necessary for continuous invasive haemodynamic monitoring and reliable blood sampling. Although no direct evidence exists in the literature, such a minimal intervention should not interfere with the hormonal effects of the anaesthetics 3 hours and 40 minutes after completion of the catheterization. This is shown by the results of $\mathrm{T}_{4}$ and noradrenaline, which returned to baseline values at the end of the study. However, it is of note that the stressful stimuli were enough to induce stress responses at $t_{1}$, albeit different responses may be expected in other surgical procedures.

\section{CONCLUSION}

The transient increase of thyroxin during surgery was not altered by the type of the anaesthetic and could not be attributed to alterations in TSH, beta-endorphin or circulating cytokines. Propofol does not seem to differ from desflurane anaesthesia in terms of the hormonal stress response, but significant differences were noted compared to sevoflurane. During surgical manipulations, propofol differed from sevoflurane in that it blunted the noradrenaline release, but it allowed for increases of ACTH and cortisol analogous to those of sevoflurane. Conversely, in the absence of surgical stimuli, propofol and sevoflurane comparably restored noradrenaline levels, but sevoflurane differed from propofol in that it resulted in increased levels of ACTH and cortisol. The results of our study clearly show that the endocrine response varies with different anaesthetics and is modified by surgery. The implications of our finding for humans are difficult to foresee and analogous studies in humans are required to clarify this issue. Nevertheless, our data offer a hint as to the selection of the most appropriate anaesthetic in either the operating room or the intensive care unit to obtain the most suitable response for a given patient or scenario.

\section{REFERENCES}

1. Desborough JP, 2000 The stress response to trauma and 
surgery. Br J Anaesth 85: 109-117.

2. Parker SD, Breslow MJ, Frank SM, et al, 1995 Catecholamine and cortisol responses to lower extremity revascularization. Correlation with outcome variables. Crit Care Med 23: 1954-1961.

3. Börner U, Klimek M, Schoengen H, Lynch J, Peschau C, Schicha H, 1995 The influence of various anesthetics on the release and metabolism of thyroid hormones: results of two clinical studies. Anesth Analg 81: 612-618.

4. Ledowski T, Bein B, Hanss R, et al, 2005 Neuroendocrine stress response and heart rate variability: a comparison of total intravenous versus balanced anesthesia. Anesth Analg 101: 1700-1705.

5. Annecke T, Kubitz JC, Kahr S, et al, 2007 Effects of sevoflurane and propofol on ischaemia-reperfusion injury after thoracic-aortic occlusion in pigs. Br J Anaesth 98: 581-590.

6. Nishiyama T, Yamashita K, Yokoyama T, 2005 Stress hormone changes in general anesthesia of long duration: isoflurane-nitrous oxide vs sevoflurane-nitrous oxide anesthesia. J Clin Anesthesia 17: 586-591.

7. Lambert A, Mitchell R, Robertson WR, 1985 Effect of propofol, thiopentone and etomidate on adrenal steroidogenesis in vitro. Br J Anaesth 57: 505-508.

8. Van Hemelrijck J, Weekers F, Van Aken H, Bouillon R, Heyns W, 1995 Propofol anesthesia does not inhibit stimulation of cortisol synthesis. Anesth Analg 80: 573576.

9. Aitkenhead AR, Pepperman ML, Willatts SM, et al, 1989 Comparison of propofol and midazolam for sedation in critically ill patients. Lancet 2: 704-709.

10. Marschall K, Schlesinger MD, Turndorf H, Puig MM, 1989 Beta-endorphin and ACTH levels in the perioperative period. Gen Pharmacol 20: 399-402.

11. Caba F, Echevarría M, Guerrero JM, Rodríguez R, 1995 Comparison of changes in plasma concentrations of ACTH and beta-endorphin in cholecystectomy under general anesthesia and general plus epidural anesthesia. Rev Esp Anestesiol Reanim 42: 364-368.

12. Wellby ML, Kennedy JA, Barreau PB, Roediger WEW, 1994 Endocrine and cytokine changes during elective surgery. J Clin Pathol 47: 1049-1051.

13. Harland WA, Horton PW, Strang R, Fitzgerald B, Richards JR, Holloway KB, 1974 Release of thyroxine from the liver during anaesthesia and surgery. Br J Anaesth 46: 818-820.

14. Kehlet H, Klauber PV, Weeke J, 1979 Thyrotropin, free and total triiodothyronine and thyroxine in plasma during surgery. Clin Endocrinol 10: 131-136.

15. Saranteas T, Tachmintzis A, Katsikeris N, et al, 2007, Perioperative thyroid hormone kinetics in patients undergoing major oral and maxillofacial operations. J Oral Maxillofac Surg 65: 408-414.

16. Chikenji T, Mizutani M, Kitsukawa Y, 1990 Anaesthesia, not surgical stress, induces increases in serum concentrations of reverse triiodothyronine and thyroxine during surgery. Exp Clin Endocrinol 95: 217-223.

17. Allaouchiche B, Debon R, Goudable J, Chassard D, Duflo F, 2001 Oxidative stress status during exposure to propofol, sevoflurane and desflurane. Anesth Analg 93: 981-985.

18. Lindgren L, Yli-Hankala A, Randell T, Kirvelä M, Scheinin M, Neuvonen PJ, 1993 Haemodynamic and catecholamine responses to induction of anaesthesia and tracheal intubation: comparison between propofol and thiopentone. Br J Anaesth 70: 306-310.

19. Pende A, Musso NR, Montaldi ML, Arzese M, Vergassola C, Devilla L, 1987 Interaction between morphine, an opioid agonist, and clonidine, an alpha-adrenergic agonist, on the regulation of anterior pituitary hormone secretion in normal male subjects. Biomed Pharmacother 41: 243-247.

20. Hangaard J, Andersen M, Grodum E, Koldkjær O, Hagen C, 1999 The effects of endogenous opioids and cortisol on thyrotropin and prolactin secretion in patients with Addison's disease. J Clin Endocrinol Metab 84: 15951601.

21. Gerard H, Sensky PL, Broom DM, Perremans S, Geers $\mathrm{R}, 1996$ Influences of type of anaesthesia on cortisol, beta-endorphin and heart rate in pigs. Vet Res 27: 219226.

22. Newman LH, McDonald JC, Wallace PG, Ledingham IM, 1987 Propofol infusion for sedation in intensive care. Anaesthesia 42: 929-937.

23. Kenyon CJ, McNeil LM, Fraser R, 1985 Comparison of the effects of etomidate, thiopentone and propofol on cortisol synthesis. Br J Anaesth 57: 509-511.

24. Fragen RJ, Weiss HW, Molteni A, 1987 The effect of propofol on adrenocortical steroidogenesis: a comparative study with etomidate and thiopental. Anesthesiology 66: 839-842.

25. Ham J, Smyth DG, 1985 Beta-endorphin and ACTH related peptides in primary cultures of rat anterior pituitary cells: evidence for different intracellular pools. FEBS Lett 190: 253-258.

26. YaDeau JT, Morelli CM, Billingsley JK, 2003 Ketamine stimulates secretion of $\beta$-endorphin from a mouse pituitary cell line. Reg Anesth Pain Med 28: 12-16.

27. Furuya K, Shimizu R, Hirabayashi Y, Ishii R, Fukuda H, 1993 Stress hormone responses to major intra-abdominal surgery during and immediately after sevoflurane-nitrous oxide anaesthesia in elderly patients. Can J Anaesth 40: 435-439.

28. Mizutani A, Hattori S, Yoshitake S, Kitano T, Noguchi T, 1998 Effect of additional general anesthesia with propofol, midazolam or sevoflurane on stress hormone levels in hysterectomy patients, receiving epidural anesthesia. Acta Anaesthesiol Belg 49: 133-139.

29. Miyawaki T, Kohjitani A, Maeda S, Kita F, Higuchi H, Shimada M, 2004 Serum cortisol level and depth of propofol-induced sedation. Acta Anaesthesiol Scand 48: 384-390. 